\title{
Selected aspects of medical care for patients with asthma and chronic obstructive pulmonary disease (COPD) in Poland
}

The study was sponsored by Polfarmex

\begin{abstract}
Introduction: Noncompliance with healthcare undoubtedly has a strong influence on the high prevalence of uncontrolled obstructive diseases. The aim of our study was to evaluate the quality of medical conduct in patients with asthma or chronic obstructive lung diseases (COPD), with encompassed two-levelled system of health care.

Material and methods: A survey of general practitioners (GP), allergists and pulmonologists practicing in Poland was performed between September and December 2016.

The basic survey included the data concerning the number of treated patients, the course of the visits, treatment regimens and whether the patients follow the instructions of the physician. The specialist survey recorded the details of the specialist visits, their frequency and character, an evaluation of the pharmacological and non-pharmacological therapies and an evaluation of the GP's actions.

Results: The basic questionnaire was completed by 807 doctors with an average of $21 \pm 9.85$ years of medical experience. Most of the interviewed individuals were GPs $(56 \%)$, followed by pulmonologists $(28 \%)$ and allergists $(16 \%)$.

The GP reported 47 cases/month with obstructive pulmonary conditions, including $48.94 \%$ asthma and $51.06 \%$ COPD patients. They diagnosed three new asthma and COPD patients per month. The allergists treated patients with asthma (105 patients/ /month), with 19 newly-diagnosed patients/month. The pulmonologists treated fewer asthma cases than COPD: 71 and 98 patients respectively. They reported 14 patients/month of newly-diagnosed COPD cases. The patients took inhaled glucocorticoids and long-acting $\beta$ adrenoceptor agonists in separate inhalers. The most frequently-used device was a disc. In opinion of the specialists, half of the therapies initiated recently by GPs for patients with asthma and COPD required modifications.

Conclusions: There is a disparity between the true state of medical care of asthma and COPD patients and globally-accepted standards.
\end{abstract}

Key words: asthma, COPD, health care

Adv. Respir. Med. 2017; 85: 179-185

\section{Introduction}

The Epidemiology of Allergic Diseases in Poland (ECAP) study reports the incidence rate for asthma in recent years in Poland to be $9.54 \%$ in adults, and $11 \%$ in children [1]. In contrast, the analogous rates in the whole of the European Union are estimated to be $8.2 \%$ among adults and $9.2 \%$ in children [2]. Chronic obstructive pulmonary disease (COPD) is most commonly caused by active rather than passive exposure to tobacco smoke, and mainly affects people aged 40 years or more. The prevalence of COPD in Poland is estimated at $9.3 \%$ of the population [3].

Although a systematic increase in the number of COPD and asthma patients was observed in the late XX century [1-3], the last decade has seen a stabilisation of this incidence rate $[4,5]$. The practical and social costs associated with these two conditions remain high, especially in regard

Address for correspondence: Prof Paweł Górski, Department of Pneumology and Allergy, $1^{\text {st }}$ Chair of Internal Medicine, Medical University of Lodz, Kopcińskiego 22, 90-153, Lodz Polska, e-mail: pawel.gorski@umed.lodz.pl

DOI: 10.5603/ARM.2017.0030

Received: 30.06 .2017

Copyright (C) 2017 PTChP

ISSN 2451-4934 
to patients with severe asthma, or those with frequent exacerbator phenotype suffering from COPD $[4,5]$. It is important to mention that according to the World Health Organisation (WHO) and the Global Initiative for chronic Obstructive Lung Disease (GOLD), COPD is also projected to become the third leading cause of death in the global population in 2020 [5].

Medical conduct, both treatment and prophylactic, is highly imperfect. This has been generally attributed to a combination of inadequate public awareness and ignorance on the part of the health care authorities. In 2013, only 3\% of adults in Poland were aware of the existence of the conditions referred to as COPD [6].

Noncompliance with healthcare is undoubtedly a serious reason behind the high prevalence of uncontrolled or incompletely controlled asthma, which was shown in 50\% of adults and $70 \%$ of children [1, 7]. Kardas et al. [8] report that 83\% of patients suffering from chronic conditions discontinue pharmacotherapy without medical consultation. The material costs of such a decision, including intermediate costs such as disabilities, are significantly higher than state expenditures associated with therapies for chronic conditions. A combined effort by the doctor, mid-level medical personnel, patient and patient's family is clearly required for effective medical care $[3,6,8-11]$. Although a wealth of literature exists which is devoted to this aim, the number of guidebooks and schools for patients and their families remains low [12]. There is limited evidence of effective training being provided for patients, or direct instruction being given by physicians: what little information is given seems to be minimally effective [13]. The knowledge possessed by the doctors and its regular updating, appear to be also imperfect [13]. Several such studies highlight the need for diligent and systematic training of the medical and paramedical personnel [2, 3, 6, 13].

One of the significant issues associated with public health is the absence of unambiguous standards concerning the diversified role of general practitioners (GP) and specialists regarding COPD. This relationship has direct implications not only on the national budget, but mainly on the effectiveness of treating individual patients [2, 3, $6,7,11]$. Therefore, the present study evaluates the quality of medical treatment provided for these groups of patients, with a two-level system of health care. It examines the significance of selected parameters which in our experience, have a particularly strong influence on the quality of medical treatment.

\section{Material and methods}

The study was performed between September 2016 and December 2016. A survey was performed of a number of general practitioners (GP), allergists and pulmonologists practising in the following Polish voivodships: Lodz, Masovia, Lesser Poland, Silesia, Lower Silesia, Kuyavia-Pomerania, West Pomerania, Lublin, Lubusz, Opole, Subcarpathia, Pomerania, Swietokrzyskie, Varmia-Masuria and Greater Poland.

The online questionnaires were completed by qualified interviewers during the course of four or six visits. All interviewers were trained for all the formal, legal and technical aspects of the research. The first visit involved the initial enrolment, with the inclusion criteria being clinicians willing to participate in the study, possessing a minimum of five years of experience in working with asthma and COPD patients, and a minimum of 10 patients with obstructive pulmonary diseases. The following three visits involved a so-called basic mode survey, which obtained data concerning the number of treated asthma patients, the course of the visits, treatment regimens and whether the patients follow the instructions. The advanced mode survey was designed for specialists experienced in chronic respiratory disorders and for allergists. The questions concerned the details of the specialist visits, their frequency, character, the evaluation of the pharmacological and non-pharmacological therapies in asthma cases, COPD and evaluation of treatment by the GP.

The data was presented as absolute values and as percentages. The central tendency was measured by arithmetic mean and median. As a measure of spread, standard deviation was used. Analysis was performed by Kantar TNS company.

\section{Results}

The basic questionnaire was completed by 807 doctors with an average of $21 \pm 9.85$ years of medical experience. Most of the interviewed individuals were GPs ( $\mathrm{n}=454 ; 56 \%)$, followed by pulmonologists ( $\mathrm{n}=223 ; 28 \%$ ), and then allergists $(\mathrm{n}=130 ; 16 \%)$. Most of those interviewed were public health care clinicians (64\%). During the following two visits, 72 allergists and 123 pulmonologists completed the advanced questionnaires. Figure 1 presents the number of participating doctors according to voivodship.

GPs reported a relatively small number of patients with obstructive pulmonary conditions: approximately 47 cases a month, including 23 
(48.94\%) asthma and 24 (51.06\%) COPD patients. They also reported typically three new diagnoses of asthma and COPD during the course of one month.

Allergists treat mainly patients with asthma (105 patients/month), with a relatively high number of newly-diagnosed individuals (19 patients/ /month). In contrast, the pulmonologists treated fewer asthma cases than COPD: 71 and 98 patients, respectively. These specialists reported a high number of newly-diagnosed COPD cases: 14 patients/month.

The survey identified comorbidities in both populations of patients (Fig. 2).

It was found that $98 \%$ of specialised departments were capable of performing spirometry including the reversibility test. However, $56 \%$ of

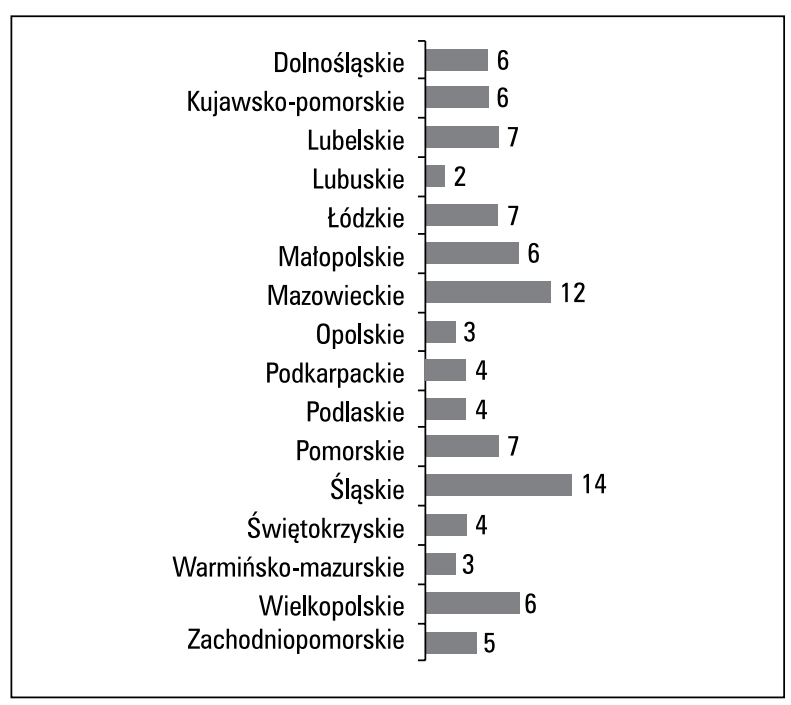

Figure 1. The number of participating doctors from particular voivodships
GP clinics was capable of performing spirometry. Only $2 \%$ of respiratory specialists reported that these tests could not be provided.

Otherwise, spirometry was reported as being performed at least once a year in patients with COPD: least commonly in GP clinics (62\%). Only $8 \%$ of asthma patients have undergone peak expiratory flow rate (PEFR) measurement. Between $12-16 \%$ of asthma patients and $9 \%$ of COPD patients discontinued medical treatment without an authorized reason (Fig. 3).

The majority of patients took the medications in separate inhalers, which included inhaled glucocorticoids (ICS) (427 patients - 26\%) and long-acting $\beta$ adrenoceptor agonists (LABA) (358 patients $-22.4 \%$ ) (Fig. 4). The most commonly-used ICS in an individual inhaler was budesonide, and the most common LABA was formoterol. The use of combined medications in a single device was marginally less common (362 patients - 22.4\%) and mainly involved salmeterol and fluticasone. Additionally, muscarinic receptor antagonist (LAMA) were used in 72 patients $(4.8 \%)$ (Table1). Inhalation treatment was most commonly (80\%) prescribed for two to three months. All three groups of respondents reported that the most frequently-used device was the disc: dry powder in multi-unit dose inhaler (Fig. 5).

The majority of allergists (97\%), pulmonologists (95\%) and GPs (83\%) declared systematic verifications of the correct use of the inhalers by the patients. During the appointments, training in the use of the inhalers was estimated to last approximately five minutes for asthma patients, and six minutes for the COPD patients. Figure 6 presents the frequency of the verifications of device usage.

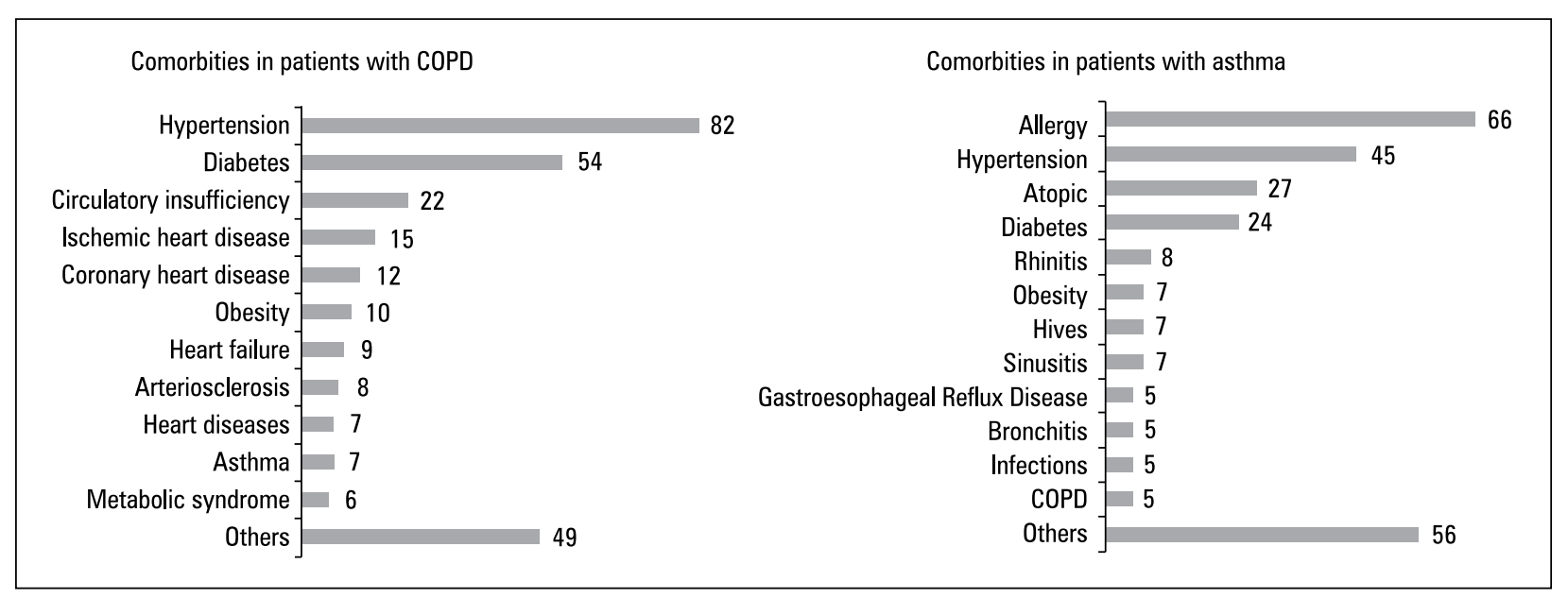

Figure 2. Comorbities in patients with COPD ad astma 


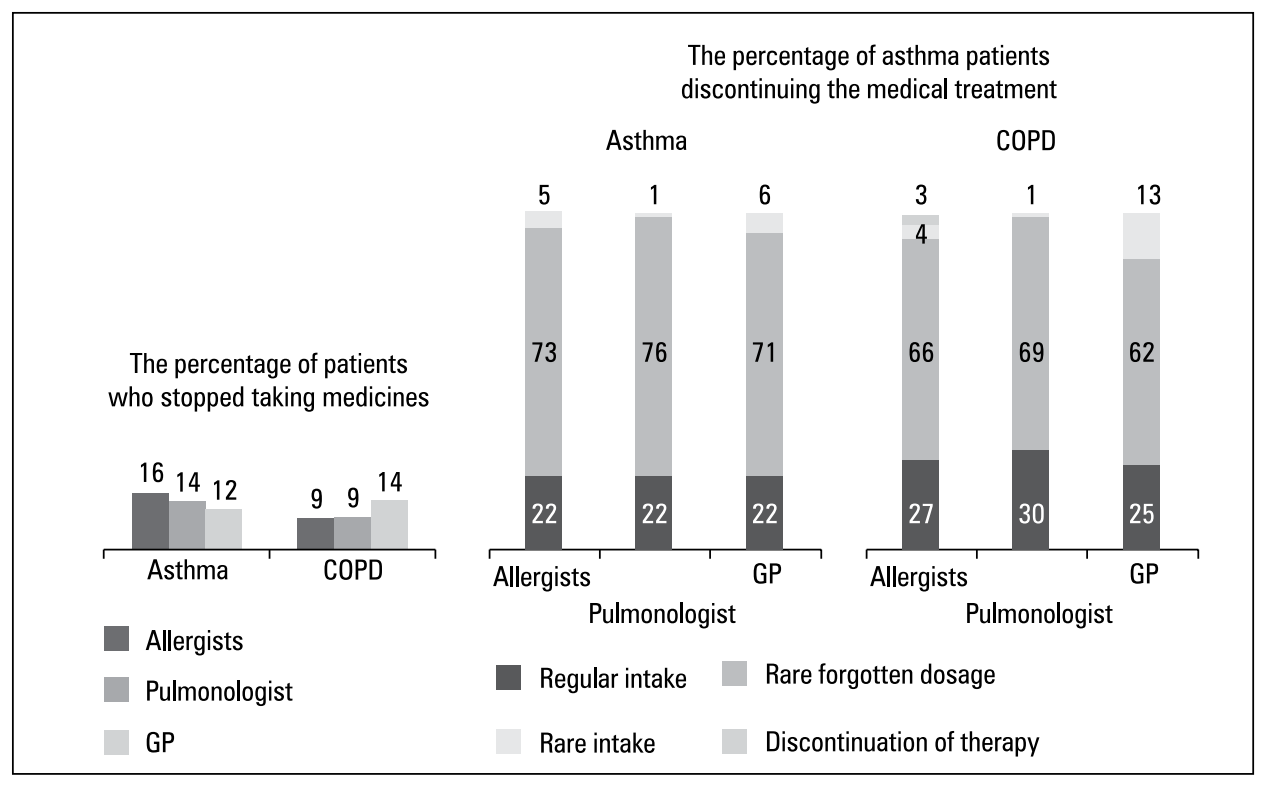

Figure 3. Compliance of the therapy according to diseases and doctors' specialization

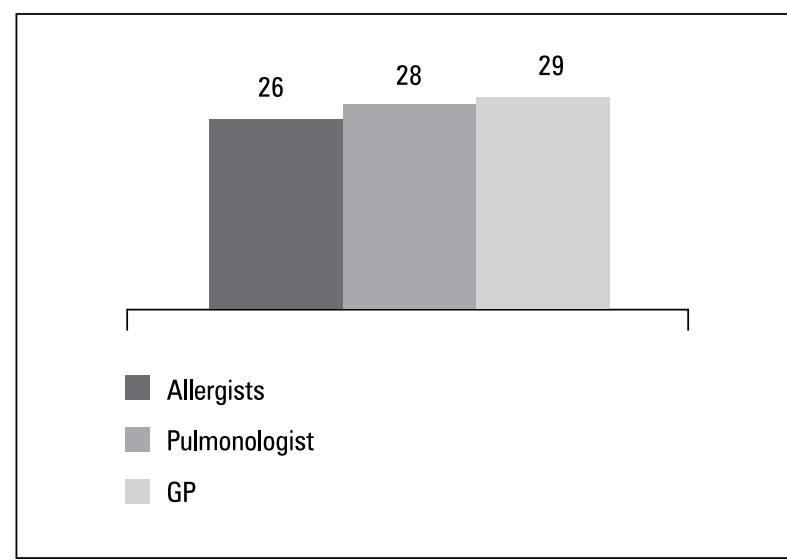

Figure 4. The percentage of patients taking two formulations in one device

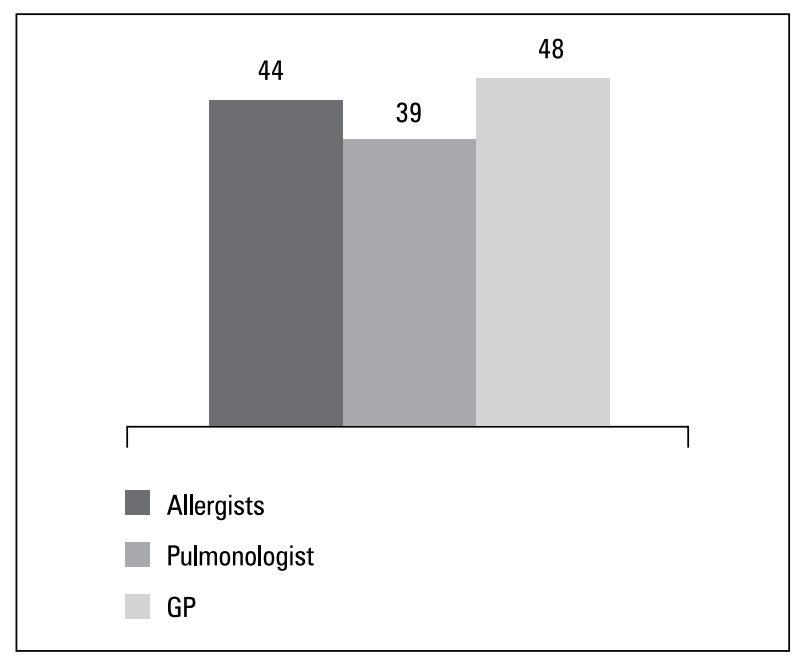

Figure 5. The percentage of patients who use disc

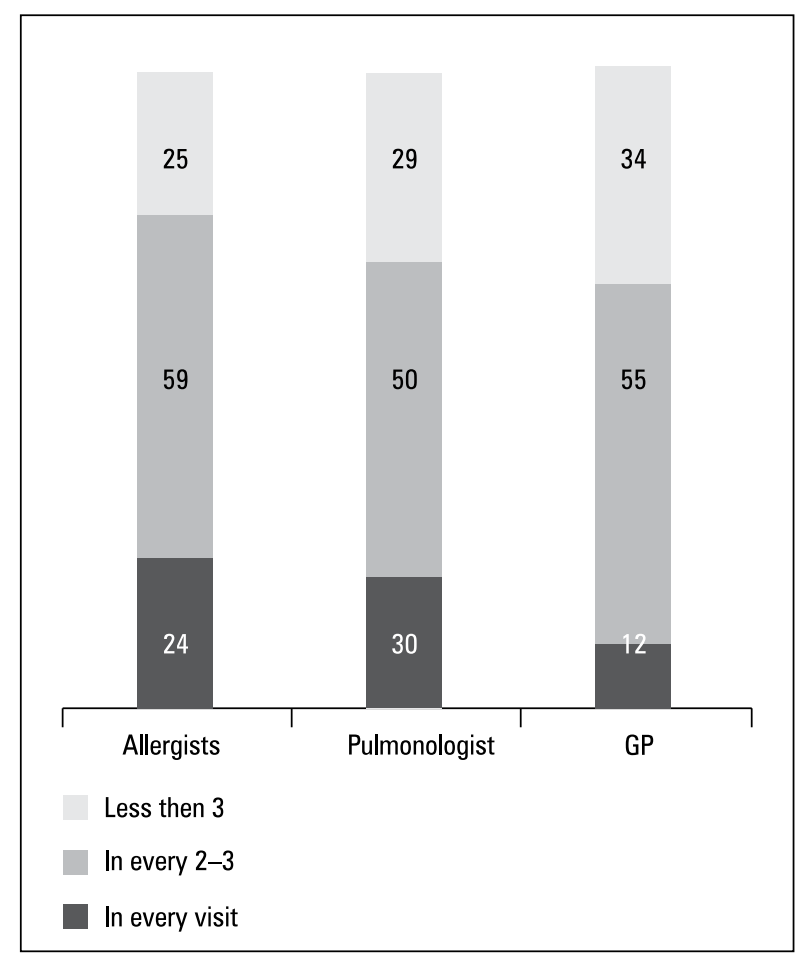

Figure 6. The frequency of control of the appropriate device usage

The results also indicated that $85 \%$ of allergists, $83 \%$ of pulmonologists and $70 \%$ of GPs stated that the nurses cooperating with them are entirely capable of delivering the appropriate instructions for the use of inhalers. Respectively, $6 \%, 11 \%$ and $14 \%$ admitted that the mid-level medical personnel they work with were not able to provide such information. Additionally, 17\% 
of allergists, $13 \%$ pulmonologists and $7 \%$ of GPs reported always asking the nurse to perform this function.

Mistakes and imperfections in the appropriate use of the medications were found in approximately $19 \%$ of cases. A small percentage of patients $(10 \%)$ were found to be using the disc inhaler incorrectly. Handouts or a demonstration device would be valuable aids to educating the patient in the correct usage of the inhaler. About $6 \%$ of the interviewees agree that internet resources or patient meetings may be helpful.

The doctors of all three groups together concluded that over $90 \%$ of their patients follow the treatment regime regularly, with only sporadic errors. The breathing exercises are applied in 15\% of COPD patients.

In opinion of the doctors specialising in respiratory conditions, half of the therapies recommended recently by GPs for patients with asthma and COPD require modifications. The specialists also report applying changes in treatment prescribed by GPs in $56 \%$ of cases. The majority of specialised doctors (88\%) provide therapeutic recommendations for GPs.

\section{Discussion}

The study gathered results from all voivodships in Poland, and so can be treated as representative of the entire country.

As the study was performed in late 2016, the conduct of the medical professionals could not be assessed with regard to the subsequently published GOLD 2017 guidelines [5]. However, it is important to highlight that the questions included in the surveys did not involve the details concerning the application of the medications, but rather the general rules governing their usage. Moreover, the guidelines do not recommend new pharmacotherapy standards for the medications used in 2010-2016 [4, 5].

In specialised clinics, obstructive pulmonary conditions are usually treated in a continuous manner. This treatment routine diverges from the official standards and actual health needs. GOLD guidelines concerning the prophylaxis and treatment of COPD, state that mild stages of the diseases and their mild exacerbations should be treated by GPs $[1,2,4,5]$. It is estimated that $85 \%$ of all asthma cases are the mild type [4]. Although detailed diagnostics and cases of exacerbations require consultation with a respiratory specialist, GPs should be able to handle mild asthma cases with appropriate basic medical conduct [4]. This disparity between the requirements and reality may account for the occurrence of undiagnosed cases and the application of inappropriate treatment $[1-11,13]$.

Some interesting results were obtained regarding testing. As the performance of spirometry tests in groups of patients at higher risk could significantly improve the identification of COPD [10], it is clear that GPs should be equipped and comprehensively educated in spirometry testing techniques. However, a disturbing finding was that $56 \%$ of surveyed clinics and $2 \%$ of specialised clinics do not perform reversibility tests, a finding that makes the validity of most diagnoses questionable.

Comorbidities identified in Polish population appear to be comparable with data obtained from global studies. Cardiovascular system disorders, diabetes, metabolic syndrome and obesity impair the general condition of the patient and worsen the prognosis of obstructive lung diseases $[4,5,9]$. Overlapping features of asthma and COPD were identified in $5 \%$ of the Polish population; however, this prevalence was found to range from 2.1 to $51 \%$ across the world, depending on study criterion and country [14].

An analysis of the general population of the Lodz voivodeship in 1998-2000 showed that $48 \%$ of adult asthma patients had discontinued the intake of anti-asthmatic medications in the previous 12 months. The individuals suffering from asthma in the previous 12 months had most commonly been prescribed LABA (46.8\%), SAMA/SABA (13\%), ICS (36.4\%), oral glucocorticoids (16.9\%), parenteral glucocorticoids (3.9\%), xanthines (33.8\%) and chromones (11.7\%) [7]: the administration of inhaled glucocorticoids was relatively low compared to the surprisingly frequent chronic administration of xanthines and chromones, suggesting that over half of the patient population received medications contrary to the official standards [4, 7].

Although another study conducted in 2016 also indicated prevalent use of ICS, it did not record xanthine or chromone use, which may suggest closer compliance with official standards [4]. It is important to note that $22.6 \%$ of interviewed doctors in the present study prescribed the use of combined inhaled glucocorticoids and LABA, which is highly recommended by global opinion-forming organisations and confirmed by several studies $[4,5,14,15]$. However, the majority of asthma cases are mild, allowing the basic application of ICS alone, with the option of dose manipulation [4]. Although our study did not include the severity of asthma, the data concerning 
the percentage of the mild asthma cases revealed doubts behind the prevalent use of the combined method [4]. Assuming that recommendations existed for the chronic use of combined inhaled glucocorticoids and LABA administration, the medications would have to be administrated together in most cases, via a single inhaler $[4,5$, 15, 16]. However, it was identified that in Poland these medications are applied separately.

The low rate of LAMA application appears to be associated partly with the difficulties associated with reimbursing this group of drugs in 2016 in Poland, and partly with the nature of previous GOLD recommendations. These recommendations were updated in February 2017, indicating the application of LAMA as one of the recommended option in cases of category A. The LAMA or LABA and LAMA/LABA combined treatment is the key of new more individualized therapy according to GOLD 2017 [5].

According to the opinion of the interviewed doctors, disc assures compliance with therapy. The disc inhaler is a medium-resistance device. Mahler et al indicates that this feature makes the inhaler especially beneficial for patients with exacerbations of COPD, resulting in a weakening of muscle strength, hyperinflation or hypoxemia. In these cases, the use of medium or low-resistance inhalers facilitates adequately low inspiratory flow and the overpowering of the resistance generated by the conglomerated particles of the medication [16]. The doctors surveyed in the present study also highlighted the user-friendly characteristics of the device, as indicated previously [17-19].

The assessment of inhaler use takes approximately five minutes in present study. Also the trainers of the Gruppo Educazionale Associazione Italiana Pneumologi Ospedalieri (AIPO) spent five minutes on instructing the patient on the correct use of the disc device and other dry powder inhalers (DPI). However, they needed significantly more time to explain the usage of the metered-dose inhaler (MDI) [19]. The training was found to extend to six minutes among doctors reporting the treatment of smaller numbers of COPD patients. The AIPO trainers reported old age as being one of the most important causes of incorrect inhalation [19]. Approximately $20 \%$ of interviewed doctors test that the device and the inhalation are correct during every visit. The majority of allergists (97\%) and pulmonologists (95\%) confirm testing in $53 \%$ of visits, i.e. every two or three visits. Over half of the patient population meet a specialist every three months. Previous studies note that patients still make mistakes even after undergoing years of inhaled therapy [19].

On the contrary, $34 \%$ of patients treated by GPs do not demonstrate the ability to use the inhaler correctly. In $38 \%$ of cases, spirometry tests are performed less than once a year. In addition, although spirometry is relatively common in specialist clinics, asthma patients rarely perform the PEFR (8\%) at home. Such management of the respiratory conditions can explain the several therapeutic decisions made by specialists to modify the treatment regimens of asthma and COPD patients initiated by the GP.

The Global Asthma Physician and Patient Survey (GAPP) conducted in 16 different countries found that approximately $40 \%$ of patients in Poland discontinue the treatment based on inhaled steroids or modify their dosage due to fear of adverse effects, without any previous consultations with attending doctors [20]. Our present findings indicate that a smaller percentage of patients discontinue treatment.

\section{Conclusion}

There is a quantitative disproportion between the true state of the medical care of asthma and COPD patients and the related globally accepted standards. Primary health care clinics lack diagnostic apparatus (spirometers), and appear to lack properly trained medical personnel skilled for identification and control of the chronic obstructive pulmonary conditions. The contribution of nurses in this regard is inadequate and does not meet the global standards.

In Poland, the most commonly-used treatment were still individual inhalers but the most commonly-used combined therapy was disc inhaler method involving salmeterol/fluticasone. The user-friendly characteristics of the device support correct inhalation of the medication, thus better control of treatment.

\section{Conflict of intrest}

The study was sponsored by Polfarmex.

\section{References:}

1. Samoliński B, Sybilski AJ, Raciborski F, et al. Prevalence of asthma in children, adolescents and young adults in Poland - results of the ECAP study. Alergia Astma Immunologia. 2009; 14(1): 27-34.

2. Selroos O, Kupczyk M, Kuna P, et al. National and regional asthma programmes in Europe. Eur Respir Rev. 2015; 24(137): 474-483, doi: 10.1183/16000617.00008114, indexed in Pubmed: 26324809 . 
3. Bednarek M, Maciejewski J, Wozniak M, et al. Prevalence, severity and underdiagnosis of COPD in the primary care setting. Thorax. 2008; 63(5): 402-407, doi: 10.1136/thx.2007.085456, indexed in Pubmed: 18234906.

4. http://ginasthma.org/. 10.06.2017.

5. http://goldcopd.org/. 10.06.2017.

6. Śliwiński P, Puchalski K. Chronic obstructive pulmonary disease in the awareness of Polish society. Report from the public opinion survey by the Polish Respiratory Society and TNS Polska. Pneumonol Alergol Pol. 2015; 83(1): 1-13, doi: 10.5603/ PiAP.2015.0001, indexed in Pubmed: 25577528.

7. Kupryś I, Elgalal A, Korzycka-Zaborowska B, et al. Korzycka-Zaborowska B. Underdiagnosis and undertreatment of asthma in general population of Lodz province (Poland), Eur Resp J. 2003; 22: 296s.

8. Kardas P. Rozpowszechnienie nieprzestrzegania zaleceń terapeutycznych wśród pacjentów leczonych z powodu wybranych schorzeń przewlekłych. Pol Merk Lek. 2011; 184: 215-220.

9. Panek M, Mokros $\mathrm{E}$, Pietras T, et al. The epidemiology of asthma and its comorbidities in Poland-Health problems of patients with severe asthma as evidenced in the Province of Lodz. Respir Med. 2016; 112: 31-38, doi: 10.1016/j. rmed.2016.01.009, indexed in Pubmed: 26852088.

10. Zielinski J, Bednarek M, Górecka $\mathrm{D}$, et al. Increasing COPD awareness. Eur Respir J. 2006; 27(4): 833-852, doi: 10.1183/09031936.06.00025905, indexed in Pubmed: 16585092.

11. Rabe KF, Vermeire PA, Soriano JB, et al. Clinical management of asthma in 1999: the Asthma Insights and Reality in Europe (AIRE) study. Eur Respir J. 2000; 16(5): 802-807, indexed in Pubmed: 11153575.

12. Hodder R, Lightstone S. Mój każdy oddech. Poradnik, jak żyć z POChP. Termedia Wydawnictwa Medyczne, Poznań 2010: wydanie II.
13. Laue J, Melbye H, Halvorsen PA, et al. How do general practitioners implement decision-making regarding COPD patients with exacerbations? An international focus group study. Int J Chron Obstruct Pulmon Dis. 2016; 11: 3109-3119, doi: 10.2147/COPD.S118856, indexed in Pubmed: 27994450.

14. Corlateanu A, Covantev S, Mathioudakis A, et al. Ashtma-Chronic obstructive pulmonary disease overlap syndrome (ACOS): current evidence and future research directions. COPD Research and Practice. 2017; 3(1), doi: 10.1186/s40749017-0025-x.

15. Rogala B, Majak P, Glück J, et al. Asthma control in adult patients treated with a combination of inhaled corticosteroids and longacting $\beta 2$ agonists: a prospective observational study. Pol Arch Intern Med. 2017; 127(2): 100-106, doi: 10.20452/ pamw.3899, indexed in Pubmed: 28220767.

16. Barnes PJ. Scientific rationale for using a single inhaler for asthma control. Eur Respir J. 2007; 29(3): 587-595, doi: 10.1183/09031936.00080306, indexed in Pubmed: 17329493.

17. Mahler D. Peak inspiratory flow rate as a criterion for dy powder inhaler use in chronic obstructive pulmonary disease. Annals of the American Thoracic Society. 2017, doi: 10.1513/ annalsats.201702-156ps.

18. Moore A, Riddell K, Joshi S, et al. Pharmacokinetics of Salbutamol Delivered from the Unit Dose Dry Powder Inhaler: Comparison with the Metered Dose Inhaler and Diskus Dry Powder Inhaler. J Aerosol Med Pulm Drug Deliv. 2017; 30(3): 164-172, doi: 10.1089/jamp.2015.1277, indexed in Pubmed: 28170282.

19. Melani AS, Bonavia M, Mastropasqua E, et al. Gruppo Educazionale Associazione Italiana Pneumologi Ospedalieri (AIPO). Time Required to Rectify Inhaler Errors Among Experienced Subjects With Faulty Technique. Respir Care. 2017; 62(4): 409-414, doi: 10.4187/respcare.05117, indexed in Pubmed: 27999149.

20. www.gappsurvey.org. 10.06.2017. 